\title{
Premenstrual syndrome and cortisol
}

\author{
Florica SANDRU ${ }^{1,2}$, Mihai Cristian DUMITRASCU ${ }^{1,3}$, Eugenia PETROVA ${ }^{1,4}$, Adina GHEMIGIAN ${ }^{1,4}$, \\ Nicoleta DUMITRU ${ }^{1,4}$, Mara CARSOTE ${ }^{1,4}$, Ana VALEA ${ }^{5,6}$ \\ 1 "Carol Davila" University of Medicine and Pharmacy, Bucharest, Romania \\ ${ }^{2}$ Elias Emergency Hospital, Bucharest, Romania \\ ${ }^{3}$ University Emergency Hospital, Bucharest, Romania \\ 4 "C.I. Parhon" National Institute of Endocrinology, Bucharest, Romania \\ 5 "Iuliu Hatieganu" University of Medicine and Pharmacy, Cluj-Napoca, Romania \\ ${ }^{6}$ Clinical County Hospital, Cluj-Napoca, Romania
}

\begin{abstract}
Premenstrual syndrome (PMS), including the severe subtype premenstrual dysphoric disorder (PMDD), DSM-5 category, represents a challenging combination of hormonal, environmental and neuroendocrine dysfunctions with menstrual cycle-related pattern. Controversies around the role of daily stress and associated anomalies of hypothalamic-pituitary-adrenal axis are related to the fact that stress is all the time, not just a fluctuating element. This is a narrative review on PMS/PMDD and cortisol profile. 46 articles are cited (between 2009 and 2020). PMD/PMDD underlines multiple imbalances and anomalies of the cortisol levels or its secretory pattern may be a few of them, despite the fact that multiple controversies are still present and most of studies are of limited statistical power. Women with PMS may have higher levels of cortisol in relationship to stress independently of the cycle phase, also a delay of CAR (cortisol awakening response) peak and a delayed cortisol slope during day time. It does not seem that CAR pattern is related to the phases of menstrual cycle. CAR anomalies may be associated with pain perception disturbances in PMS females. The most modern area of interest is related to allopregnanolone, a progesterone metabolite with neuroactive profile. The diurnal serum baseline cortisol and the values of cortisol after dexamethasone suppression test may be similar between patients with PMS and without, but the females with PMS that have higher allopregnanolone associate blunted values of cortisol during the night versus control (without PMS) and versus women with low allopregnanolone levels, thus proving a suboptimal response to stress. Allopregnanolone modules GABA receptors on a paradoxical manner inducing anxiety and irritability during luteal phase on women with a specific predisposal configuration of GABA receptor as those confirmed with PMDD. Overall, PMS/PMDD impairs the quality of life, thus the more we understand about its pathogeny, the easier it gets to control it.
\end{abstract}

Keywords: premenstrual syndrome, cortisol, premenstrual dysphoric disorder, stress, nutrition

\section{Abbreviations}

$\mathrm{CAR}=$ cortisol awakening response

GABA = acid gama-aminobutiric

GAMSA = GABAA modulating steroid antagonist

$S-\lg A=$ salivary immunoglobulin $A$
PMS = premenstrual syndrome

PMDD = premenstrual dysphoric disorder

SSRI = selective serotonin reuptake inhibitors

TSST $=$ Trier Social Stress Test 


\section{INTRODUCTION}

Premenstrual syndrome (PMS), including the severe subtype premenstrual dysphoric disorder (PMDD), DSM-5 category, represents a challenging combination of hormonal, environmental and neuroendocrine dysfunctions with menstrual cycle-related pattern (1-3). PMDD, a severe, luteal phase-related impairment of emotional and physical status with suggestive symptoms, also displays the complete remission once the menstruation is present, as well as having in common with PMS some uncertainties related to the actual underling contributor pathways like serotonin, GABA (acid gama-aminobutiric), reproductive steroids, and other brain circuits $(1,4)$. Controversies around the role of daily stress and associated anomalies of hypothalamic-pituitary-adrenal axis are related to the fact that stress is all over described in everyday life, not just a fluctuating element or menses-related and it is difficult to be quantified in specific studies of good statistics quality (5). PMS impairs the quality of life, especially PMDD (6). A combination of physical and psychiatric elements are repeating every menstrual cycle, more severe than most females of reproductive age that experience some symptoms before menstruation (up to $90 \%$ of female population having reproductive age) (6). While most of the symptoms may be controlled with lifestyle intervention, PMDD subjects are candidates to SSRI (selective serotonin reuptake inhibitors) and probably longer period of time for second line therapy with oral contraceptives in addition to alternative approaches (7).

\section{OBJECTIVE}

We aim to highlight aspects that involve PMS/ PMDD and data on cortisol levels upon on different aspects of assays.

\section{METHOD}

This is a narrative review. The research words are "premenstrual syndrome", "premenstrual dysphoric disorder" and "cortisol". 46 articles are cited (between 2009 and 2020).

\section{RESULTS}

PMD/PMDD underlines multiple hormonal imbalances, and anomalies of the cortisol levels or its secretory pattern, may be a few of them, despite the fact that multiple controversies are still present and that the most of studies are of limited statistical significance power (8). A study from 2019 on 61 women with PMDD and a similar number of control subjects evaluated subjective reactivity to stress during luteal phase in addition to assays of salivary cortisol and cortisol awakening response (CAR) showed higher levels of cortisol in relationship to stress independently of the cycle phase, a delay of CAR peak and a cortisol slope during day time which was more flatted than control (2). It has been suggested that women with PMS have a stress-related dysfunction of hypothalamic-hypophysis-adrenal axes (9). For instance, a study on 32 women with PMS (of mean age 22.47 years) versus 36 controls identified their attenuated CAR, during mid-follicular but also during late luteal phase, so it does not seem that CAR pattern is related to the phases of menstrual cycle (9). CAR anomalies may be associated with pain perception which was suggested to be disturbed in PMS females $(10,11)$. One study of 59 females with PMS (aged of 22.2 years) showed that salivary CAR increased during the premenstrual phase and not during the menses (meaning a flat CAR pattern) while salivary cortisol is positively correlated with estradiol, respective progesterone, and pain perception is more severe when ovarian steroids are low (10). A Japanese longitudinal controlled study on 31 women with PMS assessed salivary cortisol and immunoglobulin A (S-Ig A) in association with stress scores during different phased of menstrual cycle, revealing that post-menstrual S-IgA are reduced in PMS women versus control while pre-menstrual levels are higher but cortisol levels did not achieve statistical significant differences, thus S-IgA levels seem better connected with stress scales rather than cortisol in PMS women (12). Another study applied Trier Social Stress Test (TSST) on PMS subjects (13). The data on 36 females with PMS (mean age of 21.69 years) versus 36 age-matched control women revealed blunt cortisol levels as stress response according to TSST independently to the phases of menstrual cycle (13). Also, based on this study, cortisol rather than heart rate or stress scale, is a better surrogate of PMS while hypo-reactivity of hypothalamic-hypophysis-adrenal axis may be the clue of PMS severity (13). Cortisol anomalies might be the key of both emotional and cognitive processes anomalies in PMS/PMDD (14). One pilot study showed that women with PMS have increased levels of anomalies related to stress perception during luteal phase (14).

Another area of interest is related to allopregnanolone, a progesterone metabolite with neuroactive profile, which is described in another pathway, meaning that diurnal serum baseline cortisol and the values of cortisol after dexamethasone suppression test may be similar between patients with PMS and without, but the females with PMS that have higher allopregnanolone associate blunted values of cortisol during the night versus control (without PMS) and versus women with low allopregnanolone levels (15). Allopregnano- 


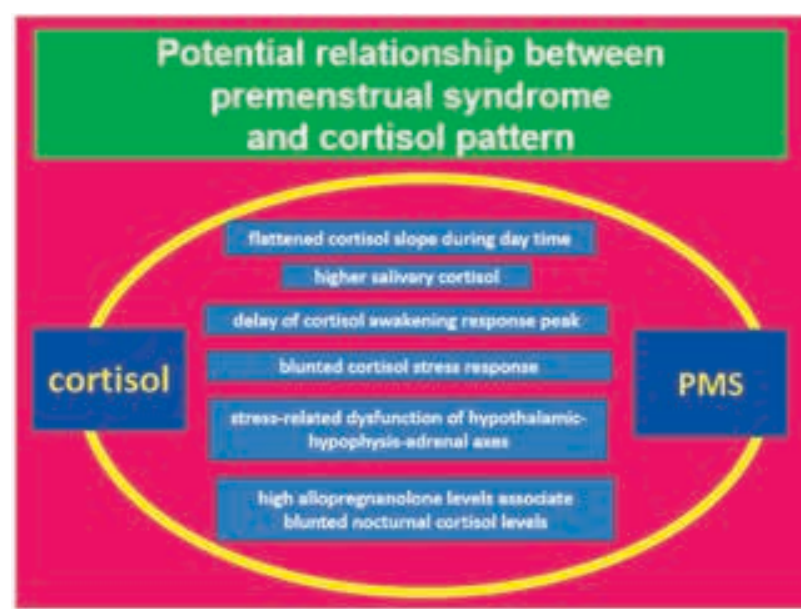

FIGURE 1. Potential relationship between premenstrual syndrome and cortisol pattern (PMS = premenstrual syndrome) (adjusted based on references 2, 8-14)

lone actually seems one of the key players of understanding the mood symptoms that appear to be due to high stress sensitivity during luteal phase (16). Allopregnanolone is a modulator of GABA receptor and in PMS females a poor control of the complex represented by GABA receptor-allopregnanolone is displayed during luteal phase under the control of hypothalamic - pituitary - adrenal axes $(16,17)$. Another collateral theory involving brain status during luteal phase of menstrual cycle may be brain inflammation (18). However, progesterone and allopregnanolone may represent useful therapy for postpartum depression, opposite to their negative role in PMS $(19,20)$. The exposure to progesterone during ovulation may increase the risk of developing PMDD $(21,22)$. Despite the fact that exact etiology of PMDD is still unknown, recently the attention was switched from cortisol to allopregnenolone and its role as modulator of GABA receptor causing sedation if the concentration is increased but paradoxically causing anxiety, irritability, depression and all the other mood elements of PMDD, if the patient with PMS has a particular configuration of GABA receptor which make her more susceptible to have the syndrome $(23,24)$. Therapy with allopregnanolone inhibitor like sepranolone which is a $\mathrm{GABA}_{A}$ modulating steroid antagonist (GAMSA) seems promising $(25,26,27)$.

Another pathogenic element, in addition to cortisol anomalies as well as allopregnanolone-GABA system anomalies, is represented by abnormal changes of serotonin pathways in PMS females $(28,29)$. That is why the first line pharmacological therapy is represented by SSRIs $(30,31)$.

Conflict of interest: none declared

Financial support: none declared

\section{DISCUSSIONS}

Other pathways that are not yet completely approved to be linked with PMS/PMDD involve hypovitaminosis $D$, otherwise with a large worldwide prevalence $(32,33)$. A meta-analysis of all databased publications until 2018 on this particular topic showed on 16 studies that 25 -hydroxyvitamin D was not correlated with PMS criteria as revealed by non-interventional studies, while interventional trials pointed that vitamin D supplementation seem efficient to some extent in controlling the cyclic symptoms, suggesting that nutrition may be a valuable approach of PMS (34). Also, calcium supplementation may be beneficial, despite the fact that dose-intervention trials are still lacking $(35,36)$. Another nutritional issue that was suggested is the level of low serum magnesium as permissive element of PMS $(37,38)$. However, not all authors agree, for instance, a meta-analysis on published papers until 2019 included 13 studies showed that there is no correlation between the level of serum magnesium and PMS symptoms during follicular, respective luteal phase (39). Another aspect is the relationship with high body mass index, obesity been another condition with a massive prevalence anywhere in the world $(40,41)$. Obesity has been associated with multiple conditions of the women within their reproductive years from fertility issues, miscarriage to urinary incontinence etc. $(42,43)$. Leptin levels, also linked with adipose tissue mass and activity, has been found higher in females with PMS based on some studies (44). However, the observation is not universal. For instance, other studies showed that high leptin is correlated with increased caloric intake in women with PMDD and high body mass index (through leptin resistance), while normal weighted females with PMDD have a decrease of leptin during late-luteal phase which is translated into overeating behaviors (45). Mood and food intake anomalies in PMDD may also be connected to dysregulation of insulin levels (46).

\section{CONCLUSION}

Overall, PMS/PMDD impairs the quality of life, especially $\mathrm{PMDD}$, thus the more we understand about its pathogeny, the easier it gets to control it. Recently, cortisol theories shifted to allopregnanolone - GABA system modulation. 


\section{REFERENCES}

1. Lanza di Scalea T, Pearlstein T. Premenstrual Dysphoric Disorder. Med Clin North Am. 2019;103(4):613-628.

2. Beddig T, Reinhard I, Kuehner C. Stress, mood, and cortisol during daily life in women with Premenstrual Dysphoric Disorder (PMDD). Psychoneuroendocrinology. 2019;109:104372.

3. Poiana C, Musat M, Carsote M, Chirita C. Premenstrual dysphoric disorder: neuroendocrine interferences. Rev Med Chir Soc Med Nat lasi. 2009;113(4):996-1000.

4. Rubinow DR, Schmidt PJ. Is there a role for reproductive steroids in the etiology and treatment of affective disorders? Dialogues Clin Neurosci. 2018;20(3):187-196.

5. Appleton SM. Premenstrual Syndrome: Evidence-based Evaluation and Treatment. Clin Obstet Gynecol. 2018;61(1):52-61.

6. Zendehdel M, Elyasi F. Biopsychosocial etiology of premenstrual syndrome: A narrative review. J Family Med Prim Care. 2018;7(2):346-356.

7. Reid RL, Soares CN. Premenstrual Dysphoric Disorder: Contemporary Diagnosis and Management. J Obstet Gynaecol Can. 2018;40(2):215-223

8. Kiesner J, Granger DA. A lack of consistent evidence for cortisol dysregulation in premenstrual syndrome/premenstrual dysphoric disorder Psychoneuroendocrinology. 2016;65:149-64.

9. Hou L, Huang Y, Zhou R. Premenstrual syndrome is associated with altered cortisol awakening response. Stress. 2019; 22(6):640-646.

10. Ozgocer T, Ucar C, Yildiz S. Cortisol awakening response is blunted and pain perception is increased during menses in cyclic women. Psychoneuroendocrinology. 2017;77:158-164.

11. Fleischman DS, Bunevicius A, Leserman J, Girdler SS. Menstrually related mood disorders and a history of abuse: moderators of pain sensitivity. Health Psychol. 2014;33(2):147-54.

12. Watanabe K, Shirakawa T. Characteristics of Perceived Stress and Salivary Levels of Secretory Immunoglobulin A and Cortisol in Japanese Women With Premenstrual Syndrome. Nurs Midwifery Stud. 2015;4(2):e24795.

13. Huang $Y$, Zhou R, Wu M, Wang Q, Zhao $Y$. Premenstrual syndrome is associated with blunted cortisol reactivity to the TSST. Stress. 2015;18(2):160-8.

14. Hoyer J, Burmann I, Kieseler ML, Vollrath F, Hellrung L, Arelin K, Roggenhofer E, Villringer A, Sacher J. Menstrual cycle phase modulates emotional conflict processing in women with and without premenstrual syndrome (PMS) - a pilot study. PLoS One. 2013 Apr 24;8(4):e59780.

15. Segebladh B, Bannbers E, Moby L, Nyberg S, Bixo M, Bäckström T, Sundström Poromaa I. Allopregnanolone serum concentrations and diurnal cortisol secretion in women with premenstrual dysphoric disorder. Arch Womens Ment Health. 2013;16(2):131-7.

16. Hantsoo L, Epperson CN. Allopregnanolone in premenstrual dysphoric disorder (PMDD): Evidence for dysregulated sensitivity to
GABA-A receptor modulating neuroactive steroids across the menstrual cycle. Neurobiol Stress. 2020 Feb 4;12:100213.

17. Pinna G. Allopregnanolone (1938-2019): A trajectory of 80 years of outstanding scientific achievements. Neurobiol Stress. 2020 Aug 5;13:100246.

18. Bannister $\mathrm{E}$. There is increasing evidence to suggest that brain inflammation could play a key role in the aetiology of psychiatric illness. Could inflammation be a cause of the premenstrual syndromes PMS and PMDD? Post Reprod Health. 2019;25(3):157-161.

19. Sundström-Poromaa I, Comasco E, Sumner $\mathrm{R}$, Luders E. Progesterone - Friend or foe? Front Neuroendocrinol. 2020 Oct;59:100856.

20. Walton N, Maguire J. Allopregnanolone-based treatments for postpartum depression: Why/how do they work? Neurobiol Stress. 2019 Oct 24;11:100198.

21. Rapkin AJ, Korotkaya Y, Taylor KC. Contraception counseling for women with premenstrual dysphoric disorder (PMDD): current perspectives. Open Access $J$ Contracept. 2019 Sep 20;10:27-39.

22. Fruzzetti F, Fidecicchi T. Hormonal Contraception and Depression: Updated Evidence and Implications in Clinical Practice. Clin Drug Investig. 2020; 40(12):1097-1106.

23. Bixo M, Johansson M, Timby E, Michalski L, Bäckström T. Effects of GABA active steroids in the female brain with a focus on the premenstrual dysphoric disorder. J Neuroendocrinol. 2018;30(2).

24. Osborn E, Wittkowski A, Brooks J, Briggs PE, O'Brien PMS. Women's experiences of receiving a diagnosis of premenstrual dysphoric disorder: a qualitative investigation. BMC Womens Health. 2020; 20(1):242.

25. Bixo M, Ekberg K, Poromaa IS, Hirschberg $A L$, Jonasson AF, Andréen L, Timby E, Wulf $M$, Ehrenborg A, Bäckström T. Treatment of premenstrual dysphoric disorder with the $\mathrm{GABA}(\mathrm{A})$ receptor modulating steroid antagonist Sepranolone (UC1010)-A randomized controlled trial. Psychoneuroendocrinology. 2017 Jun;80:46-55.

26. Bäckström T, Bixo M, Strömberg J. GABAA Receptor-Modulating Steroids in Relation to Women's Behavioral Health. Curr Psychiatry Rep. 2015 Nov;17(11):92.

27. Bäckström T, Bixo M, Johansson M, Nyberg S, Ossewaarde L, Ragagnin G, Savic I, Strömberg $\mathrm{J}$, Timby E, van Broekhoven F, van Wingen $\mathrm{G}$. Allopregnanolone and mood disorders. Prog Neurobiol. 2014 Feb;113:88-94.

28. Danis P, Drew A, Lingow S, Kurz S. Evidence-based tools for premenstrual disorders. J Fam Pract. 2020 Jan/ Feb;69(1):E9-E17

29. Yonkers KA, Simoni MK. Premenstrual disorders. Am J Obstet Gynecol. 2018; 218(1):68-74.

30. Lanza di Scalea T, Pearlstein T. Premenstrual Dysphoric Disorder. Psychiatr Clin North Am. 2017;40(2):201-216.

31. Premenstrual Syndrome and Premenstrual Dysphoric Disorder. Hofmeister S, Bodden S. Am Fam Physician. 2016 Aug 1;94(3):236-40.

32. Abdi F, Ozgoli G, Rahnemaie FS. A systematic review of the role of vitamin $D$ and calcium in premenstrual syndrome.
Obstet Gynecol Sci. 2019;62(2):73-86.

33. Jarosz AC, El-Sohemy A. Association between Vitamin D Status and Premenstrual Symptoms. J Acad Nutr Diet. 2019 Jan;119(1):115-123.

34. Arab A, Golpour-Hamedani S, Rafie N. The Association Between Vitamin D and Premenstrual Syndrome: A Systematic Review and Meta-Analysis of Current Literature. J Am Coll Nutr. 2019;38(7):648-656.

35. Arab A, Rafie N, Askari G, Taghiabadi M. Beneficial Role of Calcium in Premenstrual Syndrome: A Systematic Review of Current Literature. Int J Prev Med. 2020 Sep 22;11:156.

36. Shobeiri F, Araste FE, Ebrahimi R, Jenabi E, Nazari M. Effect of calcium on premenstrual syndrome: A double-blind randomized clinical trial. Obstet Gynecol Sci. 2017 Jan;60(1):100-105.

37. Schwalfenberg GK, Genuis SJ. The Importance of Magnesium in Clinical Healthcare. Scientifica (Cairo). 2017; 2017:4179326.

38. Parazzini F, Di Martino M, Pellegrino P. Magnesium in the gynecological practice: a literature review. Magnes Res. 2017;30(1):1-7.

39. Moslehi M, Arab A, Shadnoush M, Hajianfar $H$. The association between serum magnesium and premenstrual syndrome: a systematic review and meta-analysis of observational studies. Biol Trace Elem Res. 2019;192(2):145-152.

40. Taheri R, Mesbah Ardekani F, Raeisi Shahraki H, Heidarzadeh-Esfahani N, Hajiahmadi S. Nutritional Status and Anthropometric Indices in relation to Menstrual Disorders: A Cross-Sectional Study. J Nutr Metab. 2020 Nov 23:2020:5980685.

41. Ju H, Jones M, Mishra G. The prevalence and risk factors of dysmenorrhea. Epidemiol Rev. 2014;36:104-13.

42. Dumitrascu MC, Stanescu AMA, Bejan C, Sandru F, Toader DO, Radavoi DG, Cotirlet A, Judea Pusta CT, Diaconu CC: Obesity and its implications on stress urinary incontinence. Revista de Chimie. 2019;70(10):3660-2.

43. Dumitrascu MC, Iliescu M, Petca RC, Sandru F, Mehedintu C, Farcasanu PD, Maru N, Chibelean C, Petca A. Revista de Chimie. 2019;70(11):3818-23.

44. Nicolau ZFM, Bezerra AG, Polesel DN, Andersen ML, Tufik S, Hachul H. Plasma Leptin and Premenstrual Syndrome: A Review. Obstet Gynecol Surv. 2017;72(11):659-662.

45. Ko CH, Yen CF, Long CY, Kuo YT, Chen CS, Yen JY. The late-luteal leptin level, caloric intake and eating behaviors among women with premenstrual dysphoric disorder. Psychoneuroendocrinology. 2015;56:52-61

46. Akturk M, Toruner F, Aslan S, Altinova AE, Cakir N, Elbeg S, Arslan M. Circulating insulin and leptin in women with and without premenstrual disphoric disorder in the menstrual cycle. Gynecol Endocrinol. 2013;29(5):465-9. 